\title{
La recreación de los protagonistas del Quijote al servicio de la parodia del modernismo en el libreto de El carro de la muerte de Sinesio Delgado (1907)
}

\author{
SANTIAGO LÓPEZ NAVIA*
}

1. El carro de la muerte ${ }^{1}$, la «zarzuela fantástica extravagante» escrita en un acto único, tres cuadros y catorce escenas, cuyo libreto escribió Sinesio Delgado y a la que el maestro Tomás Barrera puso música ${ }^{2}$, se estrenó en el Teatro de la Zarzuela de Madrid en 1907 y no tenemos constancia de su reposición posterior, hecho normal sobre todo en el momento de su composición, en el que las representaciones del género eran tan frecuentes como perentorias.

En la recreación de Delgado, en cuyo texto se alternan la prosa y el verso, que sirve de letra a las canciones, se plantea una nueva resurrección de don (UNIR).

* Universidad Internacional SEK (Santiago de Chile) y Universidad Internacional de La Rioja

1. Vuelvo sobre un texto del que me ocupé por primera vez en el III Congreso Internacional de la Asociación de Cervantistas (1997) recogido luego en mi libro Inspiración y pretexto. Estudios sobre las recreaciones del Quijote (López Navia, 2005). Poco después me ocupé de la edición del texto del libreto, primero en una versión sencilla incluida en el volumen Leyendo el Quijote. IV Centenario de la publicación de Don Quijote de la Mancha editado por Ignacio Arellano en la revista Príncipe de Viana $\left(\mathrm{n}^{\mathrm{o}} 236,2005\right.$, pp. 1011-1038) y más adelante y con el necesario detalle en el volumen colectivo Don Quijote en el teatro español: del siglo de oro al siglo XX, también coordinado por Ignacio Arellano (Delgado, 2007: 535-608). Esta es la que empleo como edición de referencia en las citas del libreto de Sinesio Delgado en el presente artículo. Además de los estudios sobre la vida y la obra de Sinesio Delgado de los que doy cuenta en la bibliografía de mi edición, el lector interesado en este autor palentino, fundador de la Sociedad General de Autores y Editores, dispone de la completa tesis doctoral Bio-bibliografia de don Sinesio Delgado de José Manuel González Freire (González Freire, 2001).

2. En la obra que estudiamos, los fragmentos cantados son bastante menos significativos que los recitados, que son los más abundantes hasta el punto de que casi toda la música es incidental, y en algunos momentos forzosamente descriptiva. De hecho, don Quijote y Sancho, entre otros muchos personajes, nunca cantan, y son solo los miembros de la compañía de Silvio quienes lo hacen en determinadas ocasiones. Dada la naturaleza singular de esta zarzuela en la que el recitado prima sobre las partes cantadas, el papel de la partitura no parece especialmente significativo para reforzar la fuerza paródica inherente al texto. 
Quijote y Sancho Panza con motivo de la celebración del tercer centenario de la publicación del Quijote. En esta ocasión, la pareja de protagonistas se encuentra primero en la sierra norte de Madrid, en una zona próxima a Segovia y Ávila, con los maletillas Zoquete chico y Pupas y luego con la compañía de varietés dirigida por el singular Silvio Lilial, cuya relevancia será evidente en el objeto principal de este estudio. Nos proponemos, así, analizar los contextos en los que Sinesio Delgado parodia el alambicado estilo modernista a través de la intervención de Silvio Lilial, que se nutre de algunos de sus tópicos formales más evidentes cuya huella seguiremos en algunos textos y autores representativos del modernismo, algunos más conocidos que otros pero todos relevantes por cuanto aporta a nuestro trabajo su universo retórico.

En la obra que estudiamos, y tan fiel a su condición de caballero andante como desafortunado en sus intervenciones y en sus interpretaciones de la realidad, don Quijote fracasa en su intento de liberar a las coristas de las servidumbres de su vida artística, pero obtiene un magro triunfo al liberar a los duques de Torremormojón de la furia de los pastores de la sierra que persiguen a los protagonistas porque su extraño aspecto los asusta. En su reencuentro con la compañía de Silvio Lilial, y como consecuencia de un nuevo y disparatado error, don Quijote muere golpeado con una bandera de España por el director de las coristas ${ }^{3}$.

La confusión es la clave permanente de las peripecias del protagonista, que no entiende los verdaderos motivos de la vida de las comediantes ni la verdadera naturaleza de Silvio Lilial, a quien confunde con un encantador, ni la identidad ni el aspecto de los duques de Torremormojón, vestidos de automovilistas de los pies a la cabeza, ni la verdadera condición moral de la Ricitos, cuya vindicación inútil le lleva finalmente a la muerte. La confusión determina también los desajustes que motivan los registros de los diferentes personajes, defectivos como el argot castizo de los maletillas o como el lenguaje soez de las coristas y excesivos como los de don Quijote y Silvio Lilial, que encarnan dos estilos estridentes, uno ya hace tiempo desfasado (el caballeresco) y otro en vías de superación (el modernista), aunque en 1907 no se ha producido todavía, ni mucho menos, su declive; antes al contrario, en este momento el modernismo atraviesa un momento productivo y brillante, lo cual no lo sustrae a las críticas de sus contemporáneos. Entre ellos destaca muy activamente Emilio Ferrari, que ya había criticado en 1905, con motivo de su ingreso en la Real Academia Española, al movimiento que él consideraba como «resurrección de todas las vejeces en el Josafat de la extravagancia» (Ferrari, 1905: 6) y de cuyos tópicos y desviaciones estilísticas, de las que

3. Los personajes de la obra, tal como se enuncian en el libreto original, son la Reina del Molinete, la bella Zaida, Cucú, la Ricitos, la señora Ramona, la duquesa de Torremormojón, don Quijote de la Mancha, Sancho, el Zoquete chico, el Pupas, Silvio Lilial, el duque de Torremormojón, Bernardo, Colás y el marquesito, además de pastores, cupletistas, clowns, pierrotes, excéntricos, damas y caballeros convidados. La relación de dramatis personae se recoge en la p. 553 de la obra de referencia en la que se recoge mi edición. 
Silvio Lilial es un magnífico ejemplo, volvería a hacer sangre en su soneto burlesco «Receta para un nuevo arte» publicado tres años más tarde ${ }^{4}$.

No deja de ser curioso, sin embargo, que en el enfrentamiento (físico y dialéctico) que protagonizan estos dos personajes cuya construcción paródica ilustra las estridencias de una expresión literaria trasnochada, don Quijote considere a Silvio Lilial un «bellaco asesino del idioma» ${ }^{5}$, prueba evidente de que uno de los dos estilos parodiados se pone al servicio de la crítica del otro, en sintonía con la intención de Sinesio Delgado. Una muestra más, en fin, de la larga cadena de recreaciones del Quijote que, siglo tras siglo, han servido a sus autores para criticar, y en muchos casos ridiculizar, sistemas expresivos, posturas ideológicas o ideas religiosas.

Aunque pocas, son obvias las reminiscencias del episodio cervantino de las Cortes de la Muerte (Quijote, II, 11) tanto en la caracterización del carro de la compañía de varietés como en el recuerdo de la aventura original que conserva el Sancho de Sinesio Delgado, quien hace notar a don Quijote, siempre confundido en sus interpretaciones, que las mujeres y el carretero que vienen con él no son las princesas y el enano que acuden en su ayuda:

SANCHO Déjese de enanos y princesas, ipor los clavos de Cristo! Y más bien traiga a la memoria la famosa aventura de las cortes de la muerte, porque o mucho me engaño, o esta es otra carreta de comediantes como la que nos dio antaño aquella pesadumbre ${ }^{6}$.

A diferencia de la entusiasta percepción de don Quijote, tampoco son una garantía para Sancho las mujeres que viajan en la carreta, «pues mujeres iban también en la de marras y en lugar de luz y alegría llovieron vejigas y zambombazos» ${ }^{7}$. Sancho se refiere, desde luego, a la traumática experiencia que sufrieron los protagonistas y sus monturas en su encuentro con uno de los comediantes de la compañía de Angulo el Malo. Algo hay, quizá, de este excéntrico personaje cervantino en la caracterización y en la actitud violenta de Silvio Lilial:

Estando en estas pláticas, quiso la suerte que llegase uno de la compañía, que venía disfrazado de bojiganga, con muchos cascabeles, y en la punta de un palo traía tres vejigas de vaca hinchadas; el cual moharracho, llegándose a don Quijote, comenzó a esgrimir el palo y a sacudir el suelo

4. Aunque ninguno de los versos del soneto tiene desperdicio, baste con citar el verso final («y lengua y rima pónganse en tortura») para entender la opinión adversa que a Emilio Ferrari le merecen los artificios modernistas (Ferrari, 1908: 205).

5. Como más adelante veremos, esto ocurre en la escena XIV, justo después de que Silvio Lilial inste a don Quijote a marcharse de una forma que invitaría a cualquiera a suscribir las palabras de este entendiendo que aquel es, en efecto, un «bellaco asesino del idioma»: « ¡Huye, visión macabra, que ya basta de vesánicas elucubraciones!» (cito por mi edición, p. 600).

6. En la p. 563 de mi edición.

7. Ibidem. 
con las vejigas, y a dar grandes saltos, sonando los cascabeles; cuya mala visión así alborotó a Rocinante, que (...) dio a correr por el campo (...) Mas apenas hubo dejado su caballería Sancho por acudir a don Quijote, cuando el demonio bailador de las vejigas saltó sobre el rucio, y sacudiéndole con ellas, el miedo y el ruido, más que el dolor de los golpes, le hizo volar por la campaña ${ }^{8}$.

Queda por decir, en este encuadre de la obra de la que nos ocupamos, que El carro de la muerte se integra en el conjunto de las muchas zarzuelas que recrean el Quijote $^{9}$ a finales del siglo XIX y principios del XX y que, en términos de adscripción del texto al ámbito general de las recreaciones literarias del Quijote, el libreto forma parte de las resurrecciones, subtipo concreto y muy nutrido de continuaciones heterodoxas del original cervantino que tiene cierto éxito precisamente con motivo del tercer centenario de su publicación ${ }^{10}$.

2. Sin desdeñar en ningún momento el valor de El carro de la muerte como recreación de la obra de Cervantes, y menos aún en los años próximos a la conmemoración de su tercer centenario, nos interesa especialmente, como ya hemos dicho, su condición de texto paródico de la estética modernista. Abordamos a continuación el análisis de los contextos que ilustran de una forma más representativa esta intención.

2.1. El primero de ellos corresponde al comienzo de la escena III (cuadro primero), en el que don Quijote y Sancho se encuentran con la compañía de varietés y del que nos interesa transcribir la acotación inicial y el primer diálogo entre Silvio Lilial y las coristas:

(Aparece por el fondo izquierda todo lo siguiente: en primer lugar Silvio Lilial con frac rojo, calzón de seda, pelo lacio pegado a las sienes, monóculo grande y sombrero flexible, graciosamente arrugado. Lleva en la diestra un gran farol modernista, encendido, y en la siniestra el ronzal a que viene atado un caballejo. Este caballejo tira de un carro, también modernista, si puede ser, y en el carro están la Reina del Molinete, la bella Zaida, Cucú y la Ricitos, con trajes caprichosos y brillantes a gusto de las consumidoras. En cuanto Silvio ve a don Quijote y Sancho, hace el gesto de asombro que es de suponer y párase el carro inmediatamente.)

8. Quijote, II, 11. Cito el texto siguiendo la edición de Martín de Riquer, que refiero en la bibliografía.

9. Cito algunas de estas zarzuelas en el apartado «El marco teórico: una propuesta de los tratamientos musicales del Quijote» del capítulo «Las recreaciones musicales» de mi libro Inspiración y pretexto, ya citado (López Navia, 2005:181). Un estudio detallado de este repertorio se ofrece en el trabajo de María Encina Cortizo y Ramón Sobrino «Visiones del Quijote desde la zarzuela del siglo XX» (Encina y Sobrino, 2010: 155-202).

10. Aplico la tipología que propongo en La ficción autorial en el Quijote y en sus continuaciones e imitaciones (López Navia, 1996: 153-158). Un estudio más amplio de las obras en las que se da la resurreción del protagonista en la narrativa hispánica puede verse en mi trabajo " Contra todos los fueros de la muerte'. Las resurrecciones de don Quijote en la narrativa quijotesca hispánica» (López Navia, 2005: 67-77). 


\section{Música}

SILVIO

LAS MUJERES
Surgen de las sombras, de las sombras surgen flébiles visiones, creaciones locas, ingrávidos frutos de calenturientas imaginaciones.

Así es como Silvio expresa la aparición de los protagonistas. Nos llama la atención desde el primer momento la caracterización del personaje tanto por su atuendo, recargadamente modernista en todos sus detalles, como por su nombre, nada casual desde luego por el guiño paródico que supone su estridente apellido (Lilial), en sintonía con la denominación que los detractores del modernismo reservaban para sus adeptos, a quienes llamaban precisamente «liliales». «Poeta lilial» llama Artaza a Teófilo Pajares, el modernista que protagoniza Troteras y danzaderas de Ramón Pérez de Ayala ${ }^{12}$, y la razón es clara. Es más que sabido que el lenguaje modernista mostraba su preferencia por los lirios, como podemos constatar, entre otros muchos ejemplos posibles, por su abundancia en el poema de Rubén Darío «El poeta pregunta por Stella», incluido en Prosas profanas y otros poemas (1896-1901) (Darío, 1953: 804), y por la variante «lilios», presentes, a guisa de ejemplo, en los «lilios/del verde parque» que José María Luis Bruna, Marques de Campo, canta en el poema «Crepuscular» de su poemario Alma glau$c a$ (Luis Bruna, 1904: 30). El adjetivo «lilial» está presente, entre otros contextos, en las «manos liliales» que Rubén Darío describe en «Reino interior» (Darío, 1953: 832). Es fácil entender, a la luz de estas consideraciones, el apellido del antagonista de don Quijote.

No pasa desapercibida en la primera intervención de Silvio Lilial la preferencia del modernismo por el adjetivo «flébil» («flébiles visiones»), que aquí adquiere el sentido de triste, lamentable, lacrimoso, pero también vago e imaginario, y que el modernista mexicano Luis Gonzaga Urbina emplea en el poema «El ruiseñor cantaba» cuando se refiere al «viento flébil, alitendido y aromático» (Gimferrer, 1980: 100), pero lo que nos parece más sabroso del contexto que ahora analizamos, parodia modernista aparte, es la reacción displicente de las mujeres de la compañía, acostumbradas a las veleidades de su director y a sus incómodas consecuencias.

11. Empleo siempre mi edición, en la que el fragmento transcrito está en las páginas 564 y 565 .

12. Véase la p. 384 de la edición de Andrés Amorós en Madrid, Castalia, 1972. Agradezco a mi colega Gerardo Fernández San Emeterio la pista que me condujo a esta cita. 
2.2. El segundo fragmento que nos interesa considerar se inserta en el momento de la misma escena en que las cuatro mujeres de la compañía -la Reina del Molinete, la bella Zaida, la Ricitos y Cucú- rinden homenaje a don Quijote y Sancho tan pronto como los reconocen. Impaciente por el tiempo que pierden en el viaje las coristas, que se demoran demasiado dedicando a los protagonistas una canción y un baile sugestivos que hacen comprensible mella en sus sentimientos varoniles, Silvio Lilial llama la atención de las mujeres por su pérdida de tiempo:

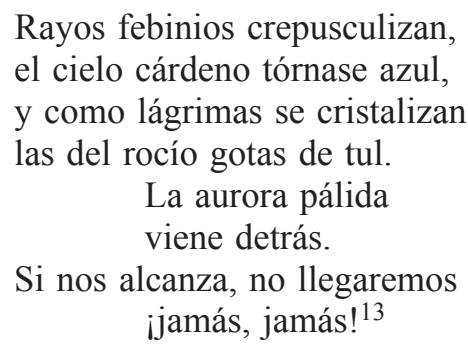

Con este estilo inequívocamente modernista da cuenta Silvio Lilial del paso del tiempo desde el crepúsculo hasta el alba: los «rayos febinios crepusculizan», o sea, los rayos de Febo, el Sol, van ganando intensidad en el momento previo al amanecer, en el que el cielo de color cárdeno se torna de color azul con la naciente luz del día que teje el manto sedoso (de tul) de las gotas del rocío, tal como Silvio canta ateniéndose a la licencia de un rotundo hipérbaton («las del rocío gotas de tul»).

La poca fuerza de los primeros rayos del sol del amanecer explica que la noche ceda ante la «aurora pálida», probable guiño a la «palidez auroral» de la que Emilio Ferrari se mofaba en su ya citada «Receta para un nuevo arte» mezclándola de nuevo con la «luz de lirio» ${ }^{14}$. Lo que le pesa a Silvio en medio de este despliegue verbal es que, si el amanecer sorprende a los viajeros en su camino, la compañía no llegará a tiempo al lugar en donde está contratada su actuación, riesgo del que él advierte a sus compañeras empleando enfáticamente el adverbio «jamás», de connotaciones trágicas bien ajustadas a las predilecciones estéticas del modernismo ${ }^{15}$.

En algún otro fragmento de estilo modernista leemos una descripción parecida, con algún matiz, del tránsito temporal que Delgado hace cantar a

13. En la p. 567 de mi edición.

14. «Disuélvanse en helénica tintura/palidez auroral y luz de lirio».

15. Llama la atención que este poético periplo que canta Silvio no parece adecuarse al momento exacto de la noche en el que se desarrolla la acción según las primeras indicaciones del cuadro primero (Monte espeso. Es de noche), teniendo en cuenta además que don Quijote y Sancho, que poco después se encuentran con la compañía, están durmiendo. Sin descartar algún descuido en un texto que, como los muchos que entonces se escribían y estrenaban, podía haberse escrito con cierta precipitación, parece evidente que Sinesio Delgado aprovecha para construir un contexto en el que los rasgos del estilo modernista que parodia le ofrecen posibilidades más jugosas. 
Silvio. Rubén Darío describe así el crepúsculo en su relato «Palomas blancas y garzas morenas», recogido en Azul (1888-1890), en el que se recrea describiendo generosamente lo que a Silvio le ocupa los dos primeros versos de su intervención:

Había un crepúsculo acariciador (...) En el cielo opalino se veía una diafanidad apacible que disminuía hasta cambiarse en tonos de violeta oscuro, por la parte del Oriente, y aumentaba convirtiéndose en oro sonrosado en el horizonte profundo, donde vibraban oblicuos, rojos y desfallecientes los últimos rayos solares (Darío, 1953: 689-690) ${ }^{16}$.

2.3. El tercer fragmento, que se inscribe en la misma parte que el anterior, corresponde al momento del diálogo entre don Quijote, la Reina del Molinete y Silvio en el que el primero, siempre confundido, expresa su agradecimiento a las coristas que han cantado para Sancho y para él poco antes y, de acuerdo con su costumbre como caballero andante, solicita a las mujeres que le digan sus nombres y el del director de la compañía, a quien confunde con un encantador que las custodia:

QUIJOTE En Dios y en mi ánima os juro, ¡oh vaporosas ninfas de esta selva!, que vuestras gracias y donosura han rendido mi fortaleza y sujeto mi ánimo. $\mathrm{Y}$ os pido y ruego que me digáis vuestros nombres y el de ese encantador que os conduce.

REINA Oye tú, Silvio; que te ha llamado encantador este caballero.

SILVIO No es el primero que me lo llama. Pero esas florículas laudantes no calman la desolación de mi vida gris y de mi alma glauca.

QUIJOTE ¿Qué ha dicho? ${ }^{17}$

La estridencia del sintagma nominal «florículas laudantes» no pasa inadvertida. Mediante esa lograda mezcla de metáfora, prosopopeya y sinestesia, todo en uno, Silvio deja claro que le concede a la palabra «encantador»-con la que don Quijote, de acuerdo con su cosmovisión caballeresca, le reconoceel valor elogioso propio de unas «florecillas de alabanza» que no alivian un sentimiento de desolación como el suyo, tan en sintonía con el taedium vitae romántico que vuelve a emerger en el modernismo y que se puede rastrear en el spleen que preside la sensibilidad simbolista de un precursor del modernismo

16. El matiz al que nos referimos tiene que ver con el momento de tiempo que refleja los instantes previos al ocaso, como hace Rubén mientras que Delgado refleja los instantes previos al alba. Recuérdese que el crepúsculo puede referirse igualmente a ambos momentos.

17. En la p. 568 de mi edición. 
tan representativo como es Baudelaire ${ }^{18}$. Como quiera que sea, las palabras de Silvio son un arcano para don Quijote, que reclama su aclaración.

Por otra parte, la sinestesia «alma glauca» se consigue con el empleo de un adjetivo que goza de las preferencias del estilo modernista a la hora de describir el color verde claro. Resulta significativo constatar que el poemario publicado en 1904 por el Marqués de Campo, ya antes citado, se titule precisamente Alma glauca. También tiene su importancia, a la hora de entender el valor que un modernista le concede a un elemento singular de su código estilístico, que el autor exponga en los preliminares de su poemario las claves simbólicas que encierra el adjetivo y que enuncian la agitación espiritual del poeta:

Ese reflejo de la onda cambiante, azul y verdoso, ese color indefinido, color glauco, turbio e indeciso, luz de pálido crisopacio, es más que ningún otro la síntesis de esos deseos infinitos, imprecisos, cambiantes y vagos, como la mirada inquietante de unas pupilas verdes (Luis Bruna, 1904: 5).

La sinestesia del título, que es literalmente la misma que emplea Silvio Lilial, no es la única del libro de José María Luis Bruna que se debe al uso del adjetivo que nos ocupa. En el poema «Remembranza!!», cuyos enfáticos signos de admiración evidencian también la vehemencia expresiva permanentemente romántica ${ }^{19}$, el poeta recuerda «esas horas tranquilas y glaucas» (Luis Bruna, 1904: 94), dando motivos a Ramiro de Maeztu para mofarse de la predilección por la sinestesia en el afectado sistema estilístico modernista en el que «se da color a las cosas que no lo tengan como besos, mirada, afecto...»" Ya con un uso descriptivo aunque fiel a sus matices simbólicos, en el poema «Green eyes» se nos dibuja un mar polícromo «glauco (...) con reflejos de rosa amarillento» (Luis Bruna, 1904: 55). Con un valor muy parecido, Rubén Darío se refiere en el relato «Palomas blancas y garzas morenas», ya citado, al «agua glauca y oscura» (Darío, 1953: 689).

2.4. El cuarto fragmento que analizamos, y que está poco después del anterior, se entiende en el momento en el que, preocupado por el tiempo precioso que están perdiendo las coristas con don Quijote y Sancho y por el retraso que puede producirse en la llegada al lugar en el que está prevista la actuación de la compañía, Silvio llama su atención instándolas a darse prisa en un diálogo que no tiene desperdicio:

18. Así es en la serie de poemas (LXXVI-LXXVIII) que desde su mismo título (Spleen) unifican el sentimiento del hastío y la desesperación. El hastío es «ese fruto de la falta de afanes» («L'ennui, fruit de la morne incuriosité», LXXVI) que siente el poeta, a quien «nadie alegrarle puede» («Rien ne peut l'égayer», LXXVII) y cuya «Esperanza, vencida/llora» («l'Espoir,/Vaincu, pleure», LXXVIII). Cito por la edición bilingüe de Las flores del mal (Baudelaire, 1993: 301-303).

19. Aunque no creo que lo que digo precise muchas aclaraciones, me atengo aquí al sentido que Guillermo Díaz-Plaja le concede al espíritu romántico como una constante de la historia de la cultura que también se hace claramente presente en el modernismo (Díaz-Plaja, 1967: 31).

20. Ramiro de Maeztu, «Poesía modernista», Los lunes del Imparcial, 14 de octubre de 1901, p.1 (Litvak, 1990: 116). 
SILVIO (Acercándose un poco.) No musitéis más. El claror opalino avanza por las espeseces del bosque y debemos ambular antes de que aurorezca.

QUIJOTE Pero, ¿qué dice?

ZAIDA Nada; que tenemos prisa. (A Silvio.) Pues anda, arrea si quieres, que ya te alcanzaremos.

SILVIO Está bien.

¡Oh banales cariátides ingratas!
¡Cómo me obstaculizan las contratas!
¡Arre, hipogrifo! (Vase con el carro por el fondo
derecha.) ${ }^{21}$

Silvio no renuncia a su modernismo trasnochado ni siquiera en momentos como este, en el que los personajes entablan un diálogo en prosa tras el anterior, en el que la canción exigía el texto en verso ${ }^{22}$. En esta nueva intervención afectada del director de la compañía, en la que el «claror opalino» sintoniza con el «cielo opalino» del fragmento antes citado del relato «Palomas blancas y garzas morenas» de Rubén Darío, la advertencia dirigida a Zaida y las demás coristas no puede ser más artificiosa: «debemos ambular antes de que aurorezca», es decir, «debemos caminar antes de que amanezca». De nuevo don Quijote, acostumbrado a suscitar en los demás la extrañeza por su discurso ajeno a lo normal, manifiesta su estupor ante el enunciado críptico de quien poco a poco dejará de ser un interlocutor para convertirse en un adversario fatal.

Los dos últimos versos en los que Silvio formula su disgusto por la indolencia y la impuntualidad de las coristas son todo un ejemplo de cómo se puede conseguir un efecto paródico a través del contraste entre el tono elevado del primero y el prosaísmo intencionado del segundo. La tópica del primer verso se nutre de las cariátides, recurrentes en la literatura modernista. Así, en el relato «La ninfa», recogido en $\mathrm{Azul}$, Rubén Darío habla de las «cariátides todas blancas y lascivas» que el protagonista de la narración puede admirar en el jardín del castillo de París en el que se desarrollan los hechos (Darío, 1953: 643), y que Amado Nervo canta («cariátides enormes, de testas milenarias») en el poema «Mi Saint Denis» del libro Poemas (1894-1900) (Nervo, 1935: 131).

2.5. En la escena XIII del cuadro tercero, después de la celebrada actuación de la bella Zaida ante el Marquesito y sus amigos en el hermoso jardín habilitado para el espectáculo, Silvio responde a la solicitud de un nuevo número por parte del público intentando recitar un poema sin demasiado éxito por

21. En la p. 571 de mi edición.

22. De lo cual no se sigue que todas las intervenciones de Silvio sean igualmente afectadas. Hay alguna excepción como los versos con los que el director de la compañía anima y cursa instrucciones a las coristas a tiempo de cantar el coro de las bayaderas ante el Marqués y sus amigos, al comienzo de la escena VIII del cuadro tercero. Sirvan como ejemplo los primeros de ellos: «Detrás de esos chopos/ estáis prevenidas,/ y al dar tres palmadas/ hacéis la salida./ ¡Mucha variedad!/ ¡Mucha exquisitez!/ Vamos a probar/ por última vez./ Una, dos, tres...». Tomo el fragmento de las pp. 583-584 de mi edición. 
causa de la airada reacción de los presentes. La situación es tan significativa como cómica:

\begin{tabular}{ll} 
OTROS & ¡Otra cosa! ¡Venga otra cosa! \\
SILVIO & $\begin{array}{c}\text { Allá voy yo. } \\
\text { «Baten el nenúfar, } \\
\text { el nenúfar baten }\end{array}$ \\
& \multicolumn{1}{c}{$\begin{array}{c}\text { ondulantes ritmos de la brisa leve...» } \\
\text { TODOS }\end{array}$} \\
(Gritando.) ¡No! ¡Que se calle! ¡Que se calle! \\
¡No! ¡No! Amigo Silvio, ¡nenúfares no! ${ }^{23}$
\end{tabular}

Aunque hay en estos versos interrumpidos algún otro motivo típicamente modernista como la «brisa leve del río», que canta Juan Ramón Jiménez en su poema «El poeta a caballo» de Baladas de primavera (1907) (Jiménez, 1980: 75), lo más llamativo es el decidido y desde luego divertido rechazo a los nenúfares, tan presentes en los textos del modernismo como para poder ahorrarnos ejemplos representativos, que implica igualmente el rechazo al estilo artificioso del movimiento y al movimiento en sí, rechazo del que participan, a su manera, las mismas coristas representadas por la Reina del Molinete, que a su debido tiempo, en la escena III, se refirió a Silvio como quien «recita versos, que no los entiende el verbo divino» ${ }^{24}$.

2.6. Por fin, a tiempo de iniciarse la galop de las banderas de la escena XIV y última de la obra, se produce la discusión final entre don Quijote y Silvio a cuenta de la reivindicación que hace el primero de la honra de la Ricitos, creyendo, en medio de su lamentable confusión, que puede convertirse en su paladín para liberarla de una servidumbre que no existe. En el primero de los dos diálogos que nos interesa reseñar, Silvio exhorta a don Quijote a retirarse empleando una adjetivación muy del gusto modernista en la que sobresale, sobre todo, la preferencia por las esdrújulas: «iHuye, visión macabra, que ya basta de vesánicas elucubraciones!» ${ }^{25}$. En el segundo, que precede al enfrentamiento que acaba con la vida de don Quijote, golpeado por la bandera de España que porta Silvio a la cabeza de la galop, la amenaza del director de la compañía adquiere un tono patético que ennoblece la respuesta del protagonista, que se arroja sobre Silvio sin poder evitar que este descargue sobre don Quijote un fuerte golpe que, unido a los empujones de los demás, acaba con su vida mientras que, herido de muerte, ruega a Sancho que le entierre envuelto en la bandera en el mismo sepulcro del que fue sacado en el tercer centenario de la publicación de la novela cervantina. Las últimas palabras del caballero son una nueva y trágica exaltación de los valores eternos que encarna el ideal que defiende, que es el mismo que, según la visión patriótica 
de Sinesio Delgado, ha amparado siempre la bandera de España que ahora le sirve de sudario:

QUIJOTE ¡Trae aquella bandera, te digo! Y vive tú que puedes, Sancho. Goza, diviértete y sacia tus apetitos como quieras, que tuyo es desde ahora el mundo. Pero muera yo; muera don Quijote de la Mancha y entiérrense con él el valor temerario y la locura sublime, el amor sin esperanzas y el dolor por el sufrimiento ajeno, el amparo de las doncellas y el socorro de los desvalidos; la fe, la generosidad y la hidalguía... ¡Cuanto ha cubierto siempre esta santa bandera ${ }^{26}$ que aquel malsín dejó en mis manos!

No es casual, y concluyo, que Silvio Lilial salpimente sus intervenciones de palabras como «flébiles», «ingrávidos», «cárdeno», «pálida», «florículas», «cariátides»y «vesánicas». A Silvio se unen entre otros, en una significativa «comunión esdrújula», el pintoresco sobrino con el que, en 1837, Ramón Mesonero Romanos hizo mofa de los románticos en su artículo «El romanticismo y los románticos», capaz de escribir un drama «emblemático-sublime, anónimo, sinónimo, tétrico y espasmódico» (Mesonero, 1845: 326-336), el ultraísta Guillermo de Torre, parodiado por Juan Manuel de Prada en Las máscaras del héroe, a quien el narrador se refiere como «aquel tarambana que hablaba con palabras esdrújulas» (Prada, 1996: 271), y el inolvidable Mendo del genial Pedro Muñoz Seca, que se refiere al Marqués de Moncada con hilarantes versos esdrújulos ${ }^{27}$. Esta comunión esdrújula, que también he

26. El espíritu de las últimas palabras de don Quijote coincide con el que preside el «Canto a la bandera» de 1906, en el que Sinesio Delgado reivindicaba el valor sagrado que encierra la bandera de España. El poema, premiado en su momento por el Ministerio de la Guerra, está recogido por Beatriz Quintana Jato (Quintana, 1999: 155). No podemos dejar de apreciar, atendiendo especialmente al papel simbólico que desempeña la bandera, una grata proximidad entre el final heroico de don Quijote en El carro de la muerte y el del personaje principal del cuento de Rubén Darío «D.Q.», publicado en el año 1899 en el Almanaque Peuser de Buenos Aires y recogido en los Cuentos fantásticos publicados en 1976 (Darío, 1976: 60-65). En esta obrita, el misterioso D.Q., abanderado de una compañía de infantería destacada en la Guerra de Cuba y de cuya identidad solo conocemos sus iniciales, se resiste épicamente a entregar la bandera a los estadounidenses actuando de un modo que invita a declinar cualquier paráfrasis en beneficio de la transcripción del fragmento original: «Cuando llegó el momento de la bandera, se vio una cosa que puso en todos el espanto glorioso de una inesperada maravilla. Aquel hombre extraño, que miraba tan profundamente con una mirada de siglos, con su bandera amarilla y roja, dándonos una mirada de la más amarga despedida, sin que nadie se atreviese a tocarle, fuese paso a paso al abismo y se arrojó en él. Todavía de lo negro del precipicio, devolvieron las rocas un ruido metálico, como el de una armadura» (Darío, 1976: 64). En su momento me ocupé de este cuento en «Dos quijotes finiseculares: 'D.Q.' de Rubén Darío» (1899) y El alma de Don Quijote, de Jerónimo Montes (1904), recogido en mi ya citado Inspiración y pretexto (López Navia, 2005: 77-90).

27. «Siempre fuisteis enigmático/ y epigramático y ático/ y gramático y simbólico,/ y aunque os escucho flemático/ sabed que a mí lo hiperbólico/ no me resulta simpático». Pedro Muñoz Seca (Muñoz Seca, 1984: 65). 
hecho notar en una cierta crítica desviada del cervantismo ${ }^{28}$ (la ambigüedad del adjetivo es deliberada), sirve para poner en evidencia, con clara intención de burla, las preferencias artificiosas y los excesos que pueden derivarse de un estilo expresivo cuya afectación puede llegar a forzar el lenguaje hasta el punto de hacerlo incomprensible. Sinesio Delgado logra su propósito con su acreditado sentido del humor y nos brinda además un texto de naturaleza metateatral y de innegable valor intrahistórico para entender la vida y la dinámica de las compañías de varietés del momento al que se adscribe la obra.

No todo es prescindible en este universo esdrújulo. Siempre nos quedarán, desde luego, los hermosos adjetivos que flanquean al sustantivo en el comienzo del evocador verso - «Ínclitas razas ubérrimas»- que Ruben Darío canta en su «Salutación del optimista» de Cantos de vida y esperanza (1905), en aposición mutua con esa «sangre de Hispania fecunda» que, en las arterias de la literatura, siente, vive y recrea el Quijote de acuerdo con lo que quieren los tiempos.

\section{BIBLIOGRAFÍA CITADA}

Baudelaire, Charles (1993). Las flores del mal, Alain Verjat y Luis Martínez de Merlo (eds.). Madrid: Cátedra.

Cervantes, Miguel de (1980). Don Quijote de la Mancha, Martín de Riquer (ed.). Barcelona: Planeta.

Cortizo, María Encina, y SoBrino, Ramón (2010). «Visiones del Quijote desde la zarzuela del siglo XX», en Begoña Lolo (ed.), Visiones del Quijote en la música del siglo $X X$. Madrid: Ministerio de Ciencia e Innovación-Centro de Estudios Cervantinos, pp. 155-202.

Darío, Rubén (1953). Obras completas. Poesía. Madrid: Afrodisio Aguado, vol. V.

Darío, Rubén (1976). Cuentos fantásticos. Madrid: Alianza Editorial.

Delgado, Sinesio (2007). El carro de la muerte, Santiago López Navia (ed.), en Ignacio Arellano (coord.), Don Quijote en el teatro español: del Siglo de Oro al siglo XX. Madrid: Visor, pp. 537-603.

Díaz Plaja, Guillermo (1967). Introducción al estudio del romanticismo español. Madrid: Espasa-Calpe.

Ferrari, Emilio (1905). Discursos leidos ante la Real Academia Española en la recepción pública de D... el día 30 de abril de 1905. Madrid: Ambrosio Pérez y Compañía, impresores.

Ferrari, Emilio (1908). Obras completas. Tomo I. Por mi camino. Poesías. Madrid: Imprenta de la Revista de Archivos.

Gimferrer, Pere (1980). Antología de la poesía modernista. Barcelona: Edicions 62.

González Freire, José Manuel (2001). Bio-bibliografía de don Sinesio Delgado (tesis doctoral). Madrid: Departamento de Filología Española IV de la Universidad Complutense de Madrid (http://eprints.ucm.es/tesis/fll/ucm-t25275.pdf).

Litvak, Lily (1990). España 1900. Barcelona: Anthropos. 
Jiménez, Juan Ramón (1980). Segunda antolojía poética. Madrid: Espasa-Calpe.

López Navia, Santiago A. (1996). La ficción autorial en el Quijote y en sus continuaciones e imitaciones. Madrid: Universidad Europea de Madrid-CEES Ediciones.

López Navia, Santiago A. (2005). Inspiración y pretexto. Estudios sobre las recreaciones del Quijote. Madrid: Iberoamericana-Vervuert.

López Navia, Santiago A. (2011). «'Sinrazones que a la razón se facen’: algunas aproximaciones esotéricas al Quijote», en Carmen Rivero Iglesias (ed.), Ortodoxia y heterodoxia en Cervantes. Alcalá de Henares: Centro de Estudios Cervantinos, pp. 329-338.

Luis Bruna, José María, Marqués de Campo (1904). Alma glauca. Madrid: Imprenta de Enrique Teodoro.

Mesonero Romanos, Ramón (1845). «El romanticismo y los románticos». Escenas matritenses. Madrid: Imprenta y librería de D. Ignacio Boix, pp. 326-336.

Muñoz Seca, Pedro (1984). La venganza de don Mendo. Madrid: Ediciones BUSMA.

Nervo, Amado (1935). Poesías completas. Madrid: Biblioteca Nueva.

Pérez de Ayala, Ramón (1972). Troteras y danzaderas, Andrés Amorós (ed.). Madrid: Castalia.

Prada, Juan Manuel (1996). Las máscaras del héroe. Madrid: Valdemar.

Quintana Jato, Beatriz (1999). Sinesio Delgado y el Madrid del 98. Palencia: Cálamo.

Recibido: 9 de noviembre de 2011

Aceptado: 20 de julio de 2012

\title{
Resumen
}

En la expresión afectada del personaje Silvio Lilial, antagonista de don Quijote en el libreto de la zarzuela de El carro de la muerte de Sinesio Delgado (1907), observamos una evidente crítica a las exageraciones del estilo modernista que supone una nueva muestra de la intención crítica (estética o ideológica) que anima a un nutrido grupo de recreaciones del Quijote. A través del estudio de los fragmentos más representativos de la intervención de Silvio, se establecen las relaciones de su forma de hablar con el universo estilístico del modernismo y se aducen, en su caso, ejemplos significativos extraídos de otros autores modernistas.

Palabras clave: Carro de la muerte; Don Quijote; modernismo; parodia; recepción; Sinesio Delgado; zarzuela.

Title: The recreation of Don Quixote's main characters at the service of parody of the modernism in the libretto of Sinesio Delgado's El carro de la muerte (1907)

\begin{abstract}
In the affected expression of Silvio Lilial, the don Quixote's antagonist in the libretto of Sinesio Delgado's El carro de la muerte (1907), we notice an evident attack on the excesses of the modernist style which represents a new sample of the critical intention (aesthetic as well as ideological) of the large group of works based in Don Quixote. Through the analysis of some of the most representative extracts of Silvio's speech, we can determine the relationship with the modernist style, considering significant examples of other modernist writers.
\end{abstract}

Key words: Carro de la muerte; Don Quixote; Modernism; Parody; Reception; Sinesio Delgado; Zarzuela. 